\title{
¿Cómo inciden en el rendimiento del juego de partidos de los niños las modificaciones del Mini Tenis de la LTA?
}

\author{
Anna Fitzpatrick, Keith Davids y Joseph Antony Stone \\ Universidad de Sheffield Hallam, Reino Unido
}

\section{RESUMEN}

Algunas versiones modificadas del tenis, como Tennis Play and Stay y Mini Tennis (MT) se implementan en todo el mundo con el fin de influir sobre las conductas y el rendimiento de los niños. Sin embargo, no queda claro cómo las versiones modificadas del tenis dan forma a las conductas durante el juego de partidos. Analizamos 1010 puntos de partido, a través de cuatro etapas (MT Rojo, MT Naranja, MT Verde y Pelota Estándar, para investigar los efectos del MT en las conductas de rendimiento durante el juego de partidos por parte de los niños (Fitzpatrick, Davids y Stone, 2017). Los peloteos con MT Rojo y MT Naranja duraron más que los jugados con Pelota Estándar, lo cual indica que el MT puede proporcionar más oportunidades para que los niños desarrollen sus destrezas. También, los jugadores de MT ejecutaron un mayor porcentaje de golpes de derecha y un menor porcentaje de golpes de revés que aquellos jugadores que lo hicieron con la pelota estándar, lo cual indica un efecto no intencional y desequilibrado que ejercen las modificaciones de la práctica sobre el desarrollo de las destrezas. Los resultados indican que los entrenadores deben considerar los efectos posibles sobre las conductas durante el juego de partidos a la hora de diseñar prácticas modificadas para jugadores junior

\author{
Palabras clave: entrenamiento \\ basado en las limitaciones, \\ escalada, compresión, mini \\ tennis \\ Recibido: 26 Nov 2017 \\ Aceptado: 09 Enero 2018 \\ Autor correspondiente: \\ Anna Fitzpatrick. Universidad \\ de Sheffield Hallam, Reino \\ Unido \\ Correo electrónico: \\ Anna.Fitzpatrick@shu.ac.u k
}

\section{INTRODUCCIÓN}

El tenis es desafiante para los jóvenes iniciantes, requiere un nivel significativo de competencia física para generar y mantener un peloteo (Farrow y Reid, 2010a). Para reducir la tasa de abandono y facilitar el desarrollo de destrezas, las federaciones han implementado versiones modificadas del deporte (ej. Tennis Play and Stay y Mini Tennis). El objetivo de estos formatos es proporcionar entornos de aprendizaje que se adapten mejor a las capacidades de los inexpertos aprendices en formación (Timmerman y cols., 2015). Por ejemplo, el Mini Tennis (MT) de la Asociación de Lawn Tenis (LTA) consta de tres etapas progresivas: MT Roja, MT Naranja y MT Verde. Las características del juego, como las dimensiones de la cancha, la compresión de la pelota y los formatos de puntaje se modifican en cada etapa, con el fin de facilitar a los niños la transición del MT al tenis estándar. Sin embargo, estas versiones modificadas del tenis se introdujeron únicamente sobre la base del conocimiento experimental y de opiniones subjetivas de los entrenadores. Es necesario contar con evaluaciones empíricas para comprender cómo afectan las habilidades de rendimiento en los niños (Larson y Guggenheimer, 2013).

Desde los comienzos del MT, según la investigación, la manipulación individual de las dimensiones de la cancha y de la presión de la pelota puede ayudar al desarrollo de las destrezas de los niños (Buszard, Reid, Masters y Farrow, 2016). Sin embargo, cuando se investiga los efectos de una única manipulación (por ej. la presión de la pelota) sobre el rendimiento, se limita la aplicación potencial práctica de los resultados, pues en $\mathrm{MT}$, se aplican simultáneamente varias modificaciones. Además, normalmente, se estudian las conductas de los niños dentro de un entorno de práctica, en lugar de examinar cómo la práctica realizada en un entorno modificado se transfiere a un contexto de juego de partidos. 
Más aún, algunos estudios (ej. Kachel, Buszard y Reid, 2015; Timmerman y cols., 2015) analizaron el rendimiento de los jugadores de nivel nacional, en lugar de hacerlo con jóvenes iniciantes inexpertos, para quienes se diseñaron originalmente las modificaciones. Por estos motivos, examinamos el rendimiento en partidos de niños de ciertas edades y de nivel de juego apropiado, a través de cuatro etapas de tenis (MTR, MTN, MTV y PE). El objetivo era comprender, si, y de qué manera, las modificaciones aplicadas en a metodología del MT afectaban las conductas de los niños durante el juego de partidos.

\section{MÉTODO}

\section{Participantes}

Se utilizaron 48 niños que se clasificaron según edades y etapa de tenis (ver Tabla 1)

\begin{tabular}{|c|c|c|c|c|}
\hline trepade tenth & $n$ & tdades (ansa) & $\begin{array}{l}\text { Eeperiencia en } \\
\text { jeega de teris }\end{array}$ & $\begin{array}{l}\text { Nimers de purtas } \\
\text { anal irades }\end{array}$ \\
\hline MTR & 15 & $3,4+-0,6$ & $2,20-\infty, 9$ & $2 y 0$ \\
\hline MTN & 16 & $8,5+-0,6$ & $3,2+-1,0$ & 253 \\
\hline MTV & 8 & $2.9 *-0.4$ & $3,3+-0,8$ & ata \\
\hline$n t$ & 6 & $137 \pi=0,5$ & $6,4+2,8$ & 140 \\
\hline
\end{tabular}

Tabla 1. Muestras de tamaños, edades y experiencia de juego (media $\pm s d$ ) cantidad de puntos analizados por etopo.

\section{Procedimiento}

Se filmó el rendimiento durante un total de 1010 puntos de partido (ver tabla 1). Los partidos se disputaron en una superficie Plexipave, con pelotas Wilson nuevas, adecuadas para cada etapa y de conformidad con las Reglas y Reglamentaciones del MT. Los datos de Video se codificaron utilizando un sistema de análisis con una "muy buena" fiabilidad entre evaluadores, $k=0.96$ (O'Donoghue, 2010). Los indicadores clave de rendimiento incluyeron los golpes de derecha, revés, juego de red y duración del peloteo (para la lista completa, ver Fitzpatrick y cols., 2017). Las variables que aparecen en la Tabla 2 fueron calculadas en Microsoft Excel.

\begin{tabular}{|c|c|}
\hline Variable dependiente & Ecuación/formula \\
\hline $\begin{array}{l}\text { Duración promedio del } \\
\text { peloteo }\end{array}$ & $\begin{array}{l}\text { duración del peloteo / número total de } \\
\text { peloteos }\end{array}$ \\
\hline$\%$ Derechas & $\begin{array}{l}\text { número de derechas / (total de todas las } \\
\text { derechas + total reveses + golpes en la zona } \\
\text { de la red) } \mathrm{x} 100\end{array}$ \\
\hline \% Revés & $\begin{array}{l}\text { número de reveses / (total de todas las } \\
\text { derechas }+ \text { total reveses }+ \text { golpes en la zona } \\
\text { de la red) } x \text { 100 }\end{array}$ \\
\hline$\%$ Juego en red & $\begin{array}{l}\text { número de juego en red / (total de todas las } \\
\text { derechas + total reveses + golpes en la ona } \\
\text { de la red) } \mathrm{x} 100\end{array}$ \\
\hline
\end{tabular}

Tabla 2. Variables para el juego de partidos.

\section{Análisis de los datos}

Para la identificación de las diferencias se analizaron los datos utilizando un análisis de variancia (ANOVA) para los datos sobre la duración del peloteo, y un diseño de ANOVA mixto para los datos sobre el tipo de golpe. Cuando se identificaron diferencias se utilizó la prueba post hoc de Gabriel (Toothaker, 1993).

\section{RESULTADOS}

Se presentan aquí dos resultados (ver todos los resultados presentados en Fitzpatrick y cols, 2017 ).

\section{Duración del peloteo}

Los resultados mostraron que los peloteos en etapa MTR (7.4 golpes) y MTN (6.6 golpes) fueron más largos que los peloteos en MTV (4.3 golpes) y pelota estándar (3.8 golpes), respectivamente. La Figura 1 muestra una progresiva disminución en la duración del peloteo a través de las diferentes etapas.

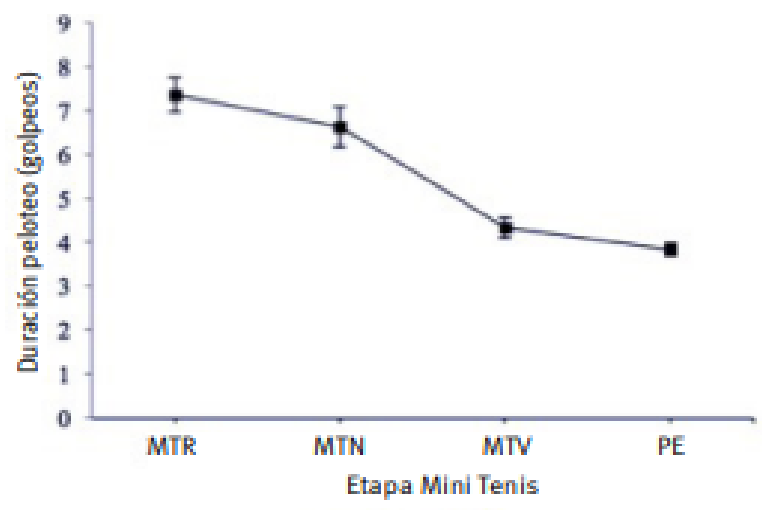

Figura 1. Duración media del peloteo en cada etapa de tenis

\section{Tipo de golpe}

Los resultados mostraron diferencias en el tipo de golpe jugado; más golpes de derecha (62.4\%) que de revés (35.0\%), y golpeos en la red (2.6\%). También hubo diferencias en el tipo de golpe que surgió en las diferentes etapas de desarrollo. La Figura 2 muestra que a medida que avanza la etapa, disminuye el porcentaje de golpes de derecha jugados: MTR (66.4\%), MTN (65.0\%), MTV (61.6\%) y PE (46.0\%); pero aumenta el porcentaje de reveses jugados: MTR (30.9\%), MTN (33.5\%), MTV (37.0\%) y PE (48.2\%). 


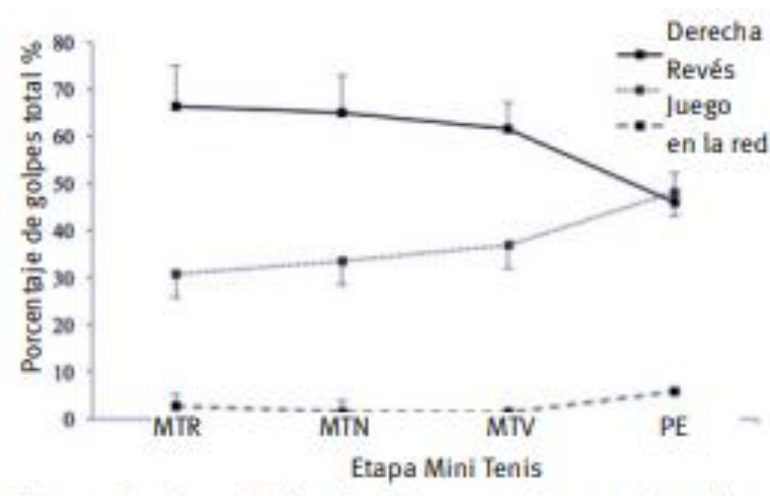

Figura 2. Desglose del tipo de golpe para cada etapa de tenis

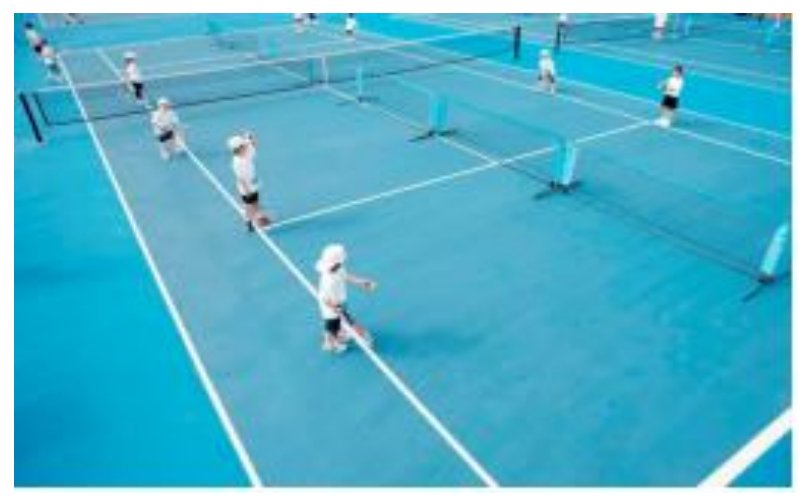

\section{COMENTARIO}

Nuestros resultados demostraron que las modificaciones de MT realmente afectan las conductas de los niños durante el juego de partidos. Cuando los partidos se juegan en canchas más pequeñas, utilizando pelotas de menor presión (MTR, por ejemplo), un resultado importante para la adquisición de habilidades fueron los peloteos más largos. Este hallazgo demuestra cómo la simplificación de la tarea puede proporcionar mayores oportunidades a los iniciantes junior para que puedan ejecutar los golpes en un entorno realista. Hubo una declinación gradual en el largo de los peloteos a medida que la tarea se tornaba más difícil (o sea, a medida que incrementaba el tamaño de la cancha y la compresión de la pelota). Nuestros resultados se asemejan a los del trabajo anterior que demostraba que escalar la cancha y modificar la pelota pueden incrementar la duración del peloteo (Farrow y Reid, 2010b). Las canchas más pequeñas reducen la distancia que el jugador debe potencialmente cubrir para alcanzar cada tiro, y las pelotas de menor compresión vuelan más lentamente y botan más bajo, de este modo, adaptan el rango de movimientos disponibles para los jugadores. En este contexto, las modificaciones de MTR proporcionan a los jugadores más tiempo para reaccionar (Martens y de Vylder, 2007) y una altura más cómoda para el contacto con la pelota, mejor alineación con la estatura física del niño, facilitando así peloteos más largos, y colaborando con su desarrollo a largo plazo (Kachel y cols., 2015).

También se identificaron diferencias entre las etapas en los tipos de golpe que surgieron. El porcentaje de golpes de derecha ejecutados disminuyó, mientras que el de reveses aumentó a medida que aumentaban las dimensiones de la cancha y la compresión de la pelota. El porcentaje relativamente alto de golpes de derecha comparado con los reveses en las tres etapas modificadas, sugiere que los jugadores prefirieron jugar el golpe de derecha más frecuentemente que de revés (aproximadamente 2:1 en la etapa MTR), en condiciones modificadas para el juego de partidos. Esta conducta puede deberse a la distancia reducida en la cual el jugador debe desplazarse y al mayor tiempo disponible para moverse alrededor de la pelota y ejecutar el golpe de derecha. Éste suele ser el primer golpe que se enseña a los niños, y por lo tanto, es el favorito. El movimiento alrededor de la pelota para ejecutar un golpe de derecha es, sin embargo, ineficiente (requiere más energía y más tiempo), con un efecto perjudicial en el momento de recuperar el centro de la cancha (Hughes y Moore, 1998). Este resultado implica también que las modificaciones del MT no proporcionan tantas oportunidades a los niños para ejecutar y desarrollar el revés como sí ocurre con el golpe de derecha. Sin embargo, la dependencia del golpe de derecha (evidente en las tres etapas de MT) disminuyó hasta la $\mathrm{PE}$, donde no se observaron diferencias entre los porcentajes de golpes de derecha y de revés ejecutados. Los trabajos anteriores sugieren que la disparidad entre los golpes de derecha y de revés puede ser aún mayor dentro de las sesiónes de entrenamiento en MT. Farrow y Reid (2010b) reportan una relación media de 6:1 a favor del golpe de derecha. Esto puede producir, con el tiempo, un desequilibrio de destrezas e inhibir el desarrollo del aprendiz. Por ejemplo, si las modificaciones MT no brindan a los niños suficientes oportunidades de ejecutar reveses, el golpe puede no desarrollarse correctamente, surgiendo así debilidades que los adversarios explotarán durante los partidos. Taylor y Hughes (1998) notaron que los jugadores adolescentes, quienes se mueven alrededor de la pelota para ejecutar un golpe de derecha, cuando un revés sería lo más indicado, cometían un número relativamente alto de errores con el revés. La importancia de desarrollar ambos golpes de fondo se apoya aún más en los datos del juego de partidos de nivel elite, que demuestran una relación golpes de derecharevés cercana a 1:1 (Reid, Morgan y Whiteside, 2016).

Es importante que los entrenadores reconozcan que la excesiva dependencia de un conjunto de modificaciones puede hacer que los participantes se vuelvan dependientes de una destreza específica y como resultado no desarrollen las otras suficientemente (por ej. el revés). Por lo tanto, es necesario contar con creatividad en el entrenamiento para diseñar las diferentes modificaciones que facilitarán las continuas adaptaciones de las destrezas por parte de los jugadores. 


\section{CONCLUSIÓN}

Nuestros resultados demostraron de qué manera el MT facilita el desarrollo de las destrezas en los niños. Sus modificaciones proporcionan a los jóvenes iniciantes mayores oportunidades de ejecutar los golpes en un entorno representativo. Como resultado se logran peloteos más largos, en canchas más pequeñas, utilizando las pelotas de baja presión. Hubo cierta disparidad entre el porcentaje de golpes de derecha y de revés realizados en las tres etapas de MT. Los entrenadores deben prestar atención a los efectos que tales modificaciones pueden causar en las conductas de los jóvenes durante el juego de partidos. Hace falta más investigación para comprobar si las adaptaciones adecuadas pueden ser aplicadas durante los entrenamientos, por aquellos entrenadores que deseen aumentar las oportunidades de lograr un mejor desarrollo de los jugadores jóvenes.

Se ha llevado a cabo un estudio de seguimiento cuyos resultados se presentarán en un futuro número de la Revista de Entrenamiento y Ciencias del Deporte de la ITF

\section{REFERENCIAS}

Buszard, T., Reid, M., Masters, R., \& Farrow, D. (2016). Scaling the equipment and play area in children's sport to improve motor skill acquisition: a systematic review. Sports Medicine, 46(6), 829843. https://doi.org/10.1007/s40279-015-0452-2

Farrow, D. \& Reid, M. (2010a). Skill acquisition in tennis, equipping learners for success. In I. Renshaw, K. Davids., \& G. J. P. Savelsbergh, (eds.), Motor learning in practice: a constraints-led approach. (pp. 231-252) Oxon: Routledge.

Farrow, D. \& Reid, M. (2010b). The effect of equipment scaling on the skill acquisition of beginning tennis players. Journal of Sports Sciences, 28(7), 723-732. https://doi.org/10.1080/02640411003770238

Fitzpatrick, A., Davids, K. \& Stone, J. A. (2017). Effects of Lawn Tennis Association Mini Tennis as task constraints on children's matchplay characteristics. Journal of Sports Sciences, 35(22), 22042210. https://doi.org/10.1080/02640414.2016.1261179

Hughes, M. \& Moore, P. (1998). Movement analysis of elite level male 'serve and volley' tennis players. In A. Lees, I. Maynard, M. Hughes and T. Reilly (eds.), Science and racket sports II. (pp. 254-259) London: E \& FN Spon.

Kachel, K., Buszard, T. \& Reid, M. (2015). The effect of ball compression on the match-play characteristics of elite junior tennis players. Journal of Sports Sciences, 33(3), 320-326. https://doi.org/10.1080/02640414.2014.942683
Larson, E. J. \& Guggenheimer, J. D. (2013). The effects of scaling tennis equipment on the forehand groundstroke performance of children. Journal of Sports Science and Medicine, 12, 323-331.

Martens, S. \& de Vylder, M. (2007). The use of low compression balls in the development of high performance players. ITF Coaches Review, 42, 3-5.

O'Donoghue, P. (2010). Research methods for sports performance analysis. (2nd ed.) Oxon: Routledge. https://doi.org/10.1080/24748668.2010.11868503 https://doi.org/10.1080/24748668.2010.11868495 https://doi.org/10.1080/24748668.2010.11868514

Reid, M., Morgan, S., \& Whiteside, D. (2016). Matchplay characteristics of Grand Slam tennis: implications for training and conditioning. Journal of Sports Sciences, 34(19), 1791-1798. https://doi.org/10.1080/02640414.2016.1139161

Taylor, M \& Hughes, M. (1998). A comparison of patterns of play between the top under 18 junior tennis players in Britain and in the rest of the world. In A. Lees, I. Maynard, M. Hughes and T. Reilly (eds.), Science and racket sports II. (pp. 260-265) London: E \& FN Spon.

Timmerman, E., de Water, J., Kachel, K., Reid, M., Farrow, D. \& Savelsbergh, G. (2015). The effect of equipment scaling on children's sport performance: the case for tennis. Journal of Sports Sciences, 33(10), 1093-1100. https://doi.org/10.1080/02640414.2014.986498

Toothaker, L. E. (1993). Multiple comparison procedures. Sage university paper series on quantitative application in the social science. Newbury Park, CA: Sage. https://doi.org/10.4135/9781412985178

CONTENIDO ITF ACADEMY RECOMENDADO (HAZ CLICK ABAJO)

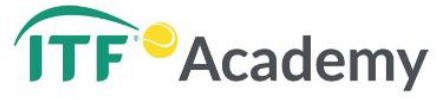

Derechos de Autor (c) 2018 Anna Fitzpatrick, Keith Davids y Joseph Antony Stone

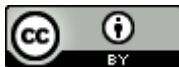

Este texto está protegido por una licencia CreativeCommons 4.0.

Usted es libre para Compartir -copiar y redistribuir el material en cualquier medio o formato- y Adaptar el documento - remezclar, transformar y crear a partir del material- para cualquier propósito, , incluso para fines comerciales, siempre que cumpla la condición de:

Atribución: Usted debe dar crédito a la obra original de manera adecuada, proporcionar un enlace a la licencia, e indicar si se han realizado cambios. Puede hacerlo en cualquier forma razonable, pero no de forma tal que sugiera que tiene el apoyo del licenciante o lo recibe por el uso que hace de la obra.

Resumendelicencia - Textocompletodelalicencia 